\title{
MR-guided motion-corrected PET image reconstruction for cardiac PET-MR*
}

\author{
Camila Munoz $^{\dagger 1}$, Sam Ellis ${ }^{\dagger 1}$, Stephan G. Nekolla ${ }^{2}$, Karl P. Kunze ${ }^{1,3}$, Teresa Vitadello ${ }^{4}$, Radhouene \\ $\mathrm{Neji}^{1,3}$, Rene M. Botnar ${ }^{1,5}$, Julia A. Schnabel ${ }^{1}$, Andrew J. Reader ${ }^{1}$ and Claudia Prieto $^{1,5}$ \\ ${ }^{1}$ School of Biomedical Engineering and Imaging Sciences, King's College London, London, UK \\ ${ }^{2}$ Nuklearmedizinische Klinik und Poliklinik, Technische Universität München, Munich, Germany \\ ${ }^{3}$ MR Research Collaborations, Siemens Healthcare, Frimley, UK \\ ${ }^{4}$ Department of Internal Medicine I, University hospital rechts der Isar, School of Medicine, Technical \\ University of Munich, Munich, Germany \\ ${ }^{5}$ Escuela de Ingeniería, Pontificia Universidad Católica de Chile, Santiago, Chile
}

First author

- Dr Camila Munoz (corresponding author)

3rd Floor, Lambeth Wing, St Thomas’ Hospital, London, SE1 7EH, UK.

Phone: +4402071888299 E-mail: camila.munoz@kcl.ac.uk

- Dr Sam Ellis

5th Floor, Becket House, 1 Lambeth Palace Road, London, SE1 7EU, UK.

Phone: +4402078489559 E-mail: $\underline{\text { sam.ellis@kcl.ac.uk }}$

Word count: 4995

*Running title: MR-guided motion-corrected cardiac PET

${ }^{\dagger}$ Contributed equally to this work

Immediate Open Access: Creative Commons Attribution 4.0 International License (CC BY) allows users to share and adapt with attribution, excluding materials credited to previous publications.

License: https://creativecommons.org/licenses/by/4.0/. Details: https://jnm.snmjournals.org/page/permissions.

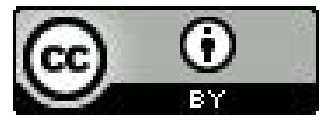




\section{ABSTRACT}

Simultaneous PET-MR imaging has shown potential for the comprehensive assessment of myocardial health from a single examination. Furthermore, MR-derived respiratory motion information has been shown to improve PET image quality by incorporating this information into the PET image reconstruction. Separately, MR-based anatomically guided PET image reconstruction has been shown to perform effective denoising, but this has been so far demonstrated mainly in brain imaging. To date the combined benefits of motion compensation and anatomical guidance have not been demonstrated for myocardial PET-MR imaging. This work addresses this by proposing a single cardiac PET-MR image reconstruction framework which fully utilises MR-derived information to allow both motion compensation and anatomical guidance within the reconstruction. Methods: Fifteen patients underwent a ${ }^{18}$ F-FDG cardiac PET-MR scan with a previously introduced acquisition framework. The MR data processing and image reconstruction pipeline produces respiratory motion fields and a high-resolution respiratory motion-corrected MR image with good tissue contrast. This MR-derived information was then included in a respiratory motion-corrected, cardiac-gated, anatomically guided image reconstruction of the simultaneously acquired PET data. Reconstructions were evaluated by measuring myocardial contrast and noise and compared to images from several comparative intermediate methods using the components of the proposed framework separately. Results: Including respiratory motion correction, cardiac gating, and anatomical guidance significantly increased contrast. In particular, myocardium-to-blood pool contrast increased by $143 \%$ on average $(p<0.0001)$ compared to conventional uncorrected, non-guided PET images. Furthermore, anatomical guidance significantly reduced image noise compared to non-guided image reconstruction by $16.1 \%(p<0.0001)$. Conclusion: The proposed framework for MR-derived motion compensation and anatomical guidance of cardiac PET data was shown to significantly improve image quality compared to alternative reconstruction 
methods. Each component of the reconstruction pipeline was shown to have a positive impact on the final image quality. These improvements have the potential to improve clinical interpretability and diagnosis based on cardiac PET-MR images.

Keywords: cardiac PET-MR, MR-guided PET reconstruction, MR-based motion correction 


\section{INTRODUCTION}

Simultaneous positron emission tomography (PET)-magnetic resonance(MR) imaging allows the comprehensive assessment of cardiovascular disease. Nearly a decade after the introduction of hybrid PET-MR systems, several studies focusing on the assessment of myocardial viability and inflammatory and infiltrative diseases have shown the benefit of the complementary functional and anatomical information provided by both imaging modalities (1-3).

PET-MR imaging has also opened new possibilities for addressing several of the technical challenges that may affect PET image quality. Accurate attenuation correction is fundamental to clinical interpretability and quantification of cardiac PET data; however, attenuation maps ( $\mu$-maps) are typically acquired under breath-hold before the actual PET acquisition, resulting in a potential misalignment between the $\mu$-map and the PET image position. This may lead to artefacts that appear as reduced myocardial uptake and could be mistaken for myocardial defects (4). To improve the correspondence between attenuation and emission data, specialised MR acquisitions schemes have been proposed to enable free-breathing (5) or respiratory-resolved $\mu$-maps (6).

Another source of image degradation is physiological (i.e. respiratory and cardiac) motion throughout PET data acquisition, which may induce blurring in the final images if not accounted for. Several approaches for MR-based PET motion compensation have been proposed, where by simultaneously acquiring dynamic MR images with sufficient tissue contrast, the organ displacement due to physiological motion can be estimated and corrected for (7-9). These techniques have shown promising results for improved PET image quality, but a limitation of most of them is that the MR images simultaneously acquired with PET data are designed for motion estimation purposes only. The insufficient spatial resolution or appropriate tissue contrast of these MR images limits their use for 
diagnostic purposes, and hinders the full realisation of the potential of truly simultaneous cardiac PETMR.

Finally, the counts-limited nature of PET image acquisition causes noise in the reconstructed images. In conventional PET image reconstruction, such as maximum likelihood expectation maximisation (MLEM) (10), this noise increases with the number of iterations, so reconstructions are usually terminated early. However, early termination results in bias due to underconvergence, reducing the quantitative value of the reconstructed PET images. In the context of PET-MR imaging, anatomical information provided by the simultaneously acquired MR images can be used to guide the PET image reconstruction, enabling noise reduction and partial volume correction $(11,12)$. Although these approaches have shown significant improvements in brain PET-MR imaging, their use in cardiac PETMR applications has not been explored so far. This is because in order to enable accurate anatomical guidance from cardiac MR images, three-dimensional images with sufficient volumetric coverage and high tissue contrast that also provide information about physiological motion (so that they can be motion-aligned to the PET image position) are required. The acquisition of such images is challenging, as most clinically available cardiac MR protocols are based on acquiring stacks of 2D slices under repeated breath-holds.

Here we introduce a single framework that exploits the advantages of hybrid cardiac PET-MR imaging to improve myocardial PET imaging, by integrating several elements of state-of-the-art PET image reconstruction. For this, we use a recently introduced cardiac PET-MR imaging protocol designed for simultaneous diagnostic PET and coronary MR angiography (CMRA) (13), which provides both respiratory motion information and a whole-heart high-resolution CMRA image that allows for myocardial PET image reconstruction to be improved as follows: (1) $\mu$-maps are aligned to the end-expiration respiratory position using the CMRA images as reference to reduce attenuation- 
induced artefacts; (2) MR-derived motion information is incorporated into a motion-corrected image reconstruction of PET data; and (3) high-contrast motion-corrected 3D CMRA images are used for anatomically guided PET image reconstruction, suppressing noise while preserving quantification performance. We tested the proposed framework in a small cohort of patients without cardiac disease to quantify the effect of each of these improvements in final image quality, including myocardium-toblood contrast and noise levels. Furthermore, we applied the framework to a cohort of ten patients with cardiac disease, showing that the proposed method achieves visually superior images compared to conventional PET image reconstruction.

\section{MATERIALS AND METHODS}

The proposed framework uses the cardiac PET-MR sequence introduced in (13) to produce high-quality whole-heart MR information that allows for accurate alignment of PET $\mu$-maps, estimation of respiratory motion fields for motion-compensated PET image reconstruction, and anatomically-guided PET image reconstruction (Figure 1). Each component of the proposed framework is described below, and a flowchart of the entire pipeline is shown in Figure 2.

\section{MR-based Respiratory Motion Correction and $\mu$-map Alignment}

The cardiac MR image reconstruction process produces 3D MR images in various respiratory states as an intermediate output, which are used to estimate respiratory-induced motion throughout the breathing cycle. This respiratory motion information can be used to correct the concurrent PET data by binning it into corresponding respiratory bins, and performing a motion-compensated PET image 
reconstruction (14) using the same motion fields as for the MR image reconstruction. Similar to the cardiac MR image, the output of this PET image reconstruction is an end-expiration cardiac PET image. To minimise the effect of cardiac motion (i.e. motion due to the heart beating), $30 \%$ of the PET data corresponding to systole are discarded using the electrocardiogram signal as a reference.

To improve the correspondence between the $\mu$-map and the PET image position, the conventional breath-held $\mu$-map is registered to the end-expiration CMRA image before PET image reconstruction. Non-rigid registration is performed using a free-form deformation algorithm with a normalised mutual information objective function (15).

\section{MR-guided PET Image Reconstruction}

The high-resolution CMRA images are then used to perform patient-specific anatomicallyguided PET image reconstructions. Anatomical guidance groups voxels which are expected to have a similar PET intensity (for example due to being in close proximity and composed of the same tissue type) and applies anisotropic smoothing between these voxels.

The proposed framework uses a weighted quadratic penalty (16), wherein the a priori similarity between a PET voxel $j$ and each of its neighbouring voxels $k$ is calculated from the MR image as the patch-based Euclidean distance modulated by a Gaussian kernel:

$$
w_{j k}=\exp \left(\frac{-\left\|\boldsymbol{f}_{j}-\boldsymbol{f}_{k}\right\|^{2}}{2 \sigma^{2}}\right) \quad \forall k \in \mathcal{N}_{j}
$$

where $\boldsymbol{f}_{j}$ is the MR patch centred at voxel $j, \mathcal{N}_{j}$ is the neighbourhood around voxel $j$, and $\sigma$ controls the width of the Gaussian similarity kernel. In this work, $\boldsymbol{f}_{j}$ are $3 \times 3 \times 3$ voxel patches, with $\mathcal{N}_{j}$ also set to $3 \times 3 \times 3$, and $\sigma=0.1$ (with MR images normalised between zero and one). Furthermore, as 
previously suggested (16), only weights corresponding to the seven most similar neighbours for each voxel are kept, with all other weights set to zero.

These similarity weights are calculated for each patient, and incorporated into the PET image reconstructions using a modified maximum a posteriori expectation maximisation (MAPEM) algorithm (17). To avoid relying on user-specified regularisation strengths, a recently proposed method for automatic setting of this value (18) was used. The regularised reconstructions were run for 200 iterations using the patient-specific automatically selected regularisation strength.

\section{Experiments}

Data acquisition. PET-MR data were acquired in 15 patients (age $62.8 \pm 12.5$ years old, 11 male, 4 female) with a simultaneous PET-CMRA sequence (13) after injection of $331.3 \pm 27.9 \mathrm{MBq}$ of ${ }^{18} \mathrm{~F}-\mathrm{FDG}$. Full details of the CMRA acquisition are given in (13).

Two patient cohorts were included in this study. The first cohort included five oncology patients without known or suspected cardiovascular disease, who exhibited physiological myocardial uptake of

${ }^{18} \mathrm{~F}$-FDG. In absence of cardiac conditions, ${ }^{18} \mathrm{~F}$-FDG uptake is expected to be uniform throughout the myocardium. These patients underwent a clinical PET-CT examination, and were subsequently scanned in a PET-MR scanner without additional radiotracer administration. This data has previously been used to demonstrate a respiratory motion compensation scheme for cardiac PET (13).

The second cohort comprised ten patients with symptomatic coronary artery disease, chronic total occlusion (CTO) of a relevant coronary artery and evidence of wall motion abnormalities. These patients underwent a clinical PET-MR examination protocol for the assessment of myocardial viability, which included the acquisition of 40-50 minutes list-mode PET acquisition using ${ }^{18} \mathrm{~F}-\mathrm{FDG}$ under insulin-clamped conditions and conventional 2D late gadolinium enhanced (LGE) MR. The data have 
previously been published in a clinical validation of respiratory motion compensation (19). Compared to previous work, the current study introduces a new PET image reconstruction framework which integrates $\mu$-map alignment, respiratory motion correction, cardiac gating and MR-guidance to further improve image quality.

All acquisitions were performed in a Biograph mMR scanner (Siemens Healthcare, Erlangen, Germany). All subjects signed a written informed consent and the study was performed in concordance with the Declaration of Helsinki and approved by the corresponding Institutional Ethics Committee.

Comparative Methods. To assess the effect of each component of the proposed cardiac PET image reconstruction method, various comparative reconstruction methods were performed:

Clinically representative reconstructions of the PET datasets were performed using MLEM (10). These reconstructions do not include respiratory motion correction (no motion-corrected, NMC), and were run for clinically representative 63 iterations (NMC-MLEM-63). To assess the effect of $\mu$-map alignment, the same reconstructions were run using $\mu$-maps registered to the end-expiration CMRA images (NMC-MLEM-63- $\mu$-reg). The NMC-MLEM reconstruction with registered $\mu$-map was also run for 200 iterations to investigate the effect of convergence (NMC-MLEM-200- $\mu$-reg).

Respiratory motion-corrected (MC) MLEM reconstructions were performed, using the registered $\mu$-map, without using anatomical guidance (MC-MLEM-200- $\mu$-reg). These reconstructions were also run to convergence, i.e. 200 iterations. To investigate the effect of cardiac motion, respiratory motion-corrected image reconstructions were performed using cardiac-gated data (MC-MLEM-200- $\mu$ reg-gated), with data acquired during systole being rejected as described above.

Finally, a reconstruction with the complete proposed method was performed, incorporating aligned $\mu$-maps, respiratory motion correction, cardiac-gating, and anatomical guidance with automatic regularisation strength selection (MC-guided-MAPEM- $\mu$-reg-gated). 
All PET image reconstructions were performed in MATLAB (Mathworks, Natick, Massachusetts, USA) with custom developed software (20). Relevant PET reconstruction parameters include resolution modelling $(4.3 \mathrm{~mm}$ full-width half maximum (FWHM)), voxel size = $2.03 \times 2.08 \times 2.08 \mathrm{~mm}^{3}$, and matrix size $=127 \times 344 \times 344$. Note that all list-mode PET data were truncated to the scan duration of the MR sequence, to allow the use of the MR respiratory trace for PET data binning.

\section{Image Analysis}

PET image quality was analysed in terms of noise and contrast. Contrast was measured as the contrast recovery coefficient (CRC) between the left-ventricular myocardium and blood pool, and noise was calculated as the standard deviation (SD) of voxels within the myocardium. To obtain the myocardial and blood pool regions, the CMRA images were semi-automatically segmented using 3DSlicer (21). To assess the local effect of motion compensation, Gaussian curves were fitted to three profiles through the left-ventricular myocardium for each oncology patient, with the estimated FWHM serving as a surrogate for myocardial sharpness. All metrics were compared between reconstruction methods with a paired 2-tailed Student $t$-test with $p<0.05$ considered a statistically significant difference.

Additionally, 17-segment analysis (22) was performed to assess the impact of the proposed method in quantification at a segment-level, and contrast between healthy myocardium and myocardial viability defects was computed from manually defined regions of interest in patients with transmural defects. 


\section{RESULTS}

Figure 3 shows the effect of each element of the proposed method in terms of CRC and myocardium SD for the first patient cohort, while Figure 4 demonstrates these differences in the reconstructed images for two representative patients.

Correcting PET $\mu$-maps was seen to have no strong impact on myocardium SD in any of the patients. In terms of CRC, four of the five patients showed no change, however, in one case (P1), an increase in CRC is apparent. This is due to a particularly poor alignment between the average freebreathing end-expiration position and the position of the breath-hold $\mu$-map, leading to a defectmimicking artefact which is alleviated by aligning the $\mu$-map (cyan arrows in Figure 4).

With a correctly aligned $\mu$-map, increasing the number of MLEM iterations from 63 to 200 was seen to increase myocardium SD and CRC in all cases. These effects are due to the convergence of the MLEM algorithm; improved contrast represents convergence towards true regional means, while increased noise at convergence is a well-known characteristic of MLEM reconstructions.

When including motion correction in the form of MC-MLEM-200- $\mu$-reg, contrast and noise both increase further due to the deblurring effect of motion correction (blue arrows in Figure 4), and increased noise arising from the MC-MLEM algorithm, as has been previously shown in the literature $(7,23,24)$. Performing cardiac gating of PET images using MC-MLEM-200- $\mu$-reg-gated greatly increases myocardial CRC, due to the removal of blurring artefacts from cardiac motion. However, noise also increases since reducing counts from the PET data (by rejecting systolic data) results in a lower signal-to-noise-ratio. Finally, by including MR-guidance in the motion-corrected, cardiac-gated PET reconstruction, CRC generally remains at similar or slightly higher levels while noise reduces. 
Similar trends were observed for the CTO patients. Figure 5 shows example PET images reconstructed with the conventional NMC-MLEM-63, MC-MLEM-200- $\mu$-reg-gated, and the proposed MC-guided-MAPEM- $\mu$-reg-gated methods alongside reference LGE MR images highlighting regions of myocardial scar. The previously described improvements in terms of noise and contrast are visible, and the depiction of myocardial defects (hypo-intense regions, cyan arrows) is preserved, coinciding with the hyper-intense regions in the LGE MR images.

The proposed MC-guided-MAPEM- $\mu$-reg-gated method increases the CRC of transmural defects by $18.6 \%$ and their contrast-to-noise ratio by $47.7 \%$ (Supplementary Figure 1), indicating that the MR guidance information is sufficiently localised to maintain relevant patterns of uptake while successfully reducing noise.

Summary statistics for both CRC and SD for all patients are shown in Table 1. Each incremental improvement in the PET image reconstruction method increased CRC significantly, except for when introducing motion correction $(\mathrm{p}=0.078)$. For myocardial SD, all differences were significant, except for alignment of the $\mu$-map ( $\mathrm{p}=0.20$ ). The only comparison in which SD decreased significantly was when introducing MR-guidance to the MC-MLEM reconstruction. Furthermore, 17-segment analysis indicates that all segments show an increased uptake with the proposed method, which ranges from $\sim 15 \%$ increase at the apical segments to over $60 \%$ increase towards the basal anterior segments (Supplementary Figure 2).

In terms of myocardial sharpness, a similar trend can be observed (Supplementary Table 1), with the proposed MC-guided-MAPEM- $\mu$-reg-gated method reducing FWHM by $24.6 \pm 13.9 \%$ compared to the conventional NMC-MLEM-63, and MC-MLEM-200- $\mu$-reg-gated images reducing FWHM by $26.6 \pm 16.9 \%$, with no statistically significant difference between both methods $(p=0.27)$. 


\section{DISCUSSION}

The aim of this study was to introduce a framework for motion-corrected, MR-guided PET image reconstruction for myocardial PET-MR imaging and demonstrate the capabilities of the proposed method to improve PET image quality by increasing contrast without introducing excessive noise into the output images.

A series of incrementally improved PET image reconstruction approaches were compared for two cohorts of patients, with and without known cardiac disease. Quantitative showed that each of the incremental improvements increased contrast (Figure 3), however, this was generally at the cost of additional noise. Compared to the MC-MLEM-200- $\mu$-reg-gated reconstruction, the proposed method incorporating MR-guidance further improved contrast while reducing noise. Statistical analysis showed that these improvements were generally statistically significant, with the notable exception of the respiratory motion-corrected reconstruction.

This lack of significance when including motion correction may be due to both the non-rigid nature of the respiratory-induced motion of the heart and the variability in respiratory patterns across different patients. While motion correction can demonstrate strong improvement in areas of high motion (e.g. Figure 4, blue arrows), areas of reduced motion in patients with shallower breathing patterns will result in less difference between corrected and uncorrected images.

It is worth noting that the proposed method was not observed to reduce noise down to clinical standard levels (Table 1). However, noise levels in clinical PET images (i.e. at 63 iterations) are arbitrary since sufficiently early termination can provide almost arbitrarily low noise. Similarly, the proposed method could achieve additional noise reduction by manually varying the regularisation strength. This manually selected regularisation would still lead to increased CRC at matched noise 
levels, although a comprehensive evaluation of this approach remains for future work. Furthermore, the automatic regularisation selection method is designed to be error-optimal, rather than noise-optimal (18). When the guidance information is imperfect, regularisation strength is limited to produce a faithful, if more noisy representation of the PET data. For this reason, the noise levels from the proposed reconstruction method are a function of both raw PET data noise level and accuracy of correspondence between the MR and PET images. By producing better MR-based guidance information, or modelling uncertainties of guidance information, regularisation strength could be increased, further reducing noise.

\section{Limitations and Future Work}

Despite the promising results demonstrated in this study, there are several areas for improvement that could be addressed in future work.

The proposed reconstruction method requires the selection of a number of tuneable hyperparameters, including motion estimation (e.g. number of respiratory bins, image registration parameters) and anatomical guidance parameters (e.g. $\mathcal{N}_{j}, \sigma$ ). In this work, such parameters were selected as of previous studies, and their impact in the final image quality was not studied. Future work includes a comprehensive investigation of these hyperparameters, which would be required before using the proposed method in a clinical setting. Furthermore, the proposed reconstruction is slow, requiring $\sim 10$ hours of computing time per patient, using a single Intel Xeon $2.6 \mathrm{GHz} \mathrm{CPU}$ with an NVIDIA Tesla K40M GPU used for the PET projection operators, which is impractical for clinical adoption. Options for acceleration include pursuing a subsets-based implementation (25), or applying the MR-guidance post-reconstruction with a deep-learning approach (26). 
While this study demonstrated improvements in terms of contrast and noise considering the whole myocardium, and highlighted correspondence between PET and LGE images, the clinical utility of the images produced by the proposed method in terms of detection and assessment of myocardial defects was not thoroughly evaluated, although preliminary assessment showed the proposed method increased the contrast-to-noise ratio for transmural defects. Further studies where the detectability and delineation of myocardial viability defects are assessed by expert observers in a larger cohort of patients are required to fully assess the diagnostic value of this technique.

In clinical practice, cardiac PET-MR protocols can be up to 40 minutes long, but the proposed framework uses only $\sim 10$ minutes of simultaneous PET-CMRA data. The method could be extended to include longer PET acquisition durations, by using surrogate respiratory motion signals (8).

Although the PET and MR data in this study are simultaneously acquired, there remains no guarantee that the positions of the two images correspond perfectly. System imperfections could lead to misalignment between imaging modalities. Additionally, the diastolic PET acquisition window in each heartbeat is much longer than the MR acquisition window, potentially leading to residual cardiac motion in the PET data that could cause misalignment between the PET and MR images. This could be alleviated by gating the PET data even more restrictively. However, this would reduce the counts in the PET data further, potentially impacting image quality. Alternatively, this cardiac-induced misalignment could be estimated during the reconstruction (27), although this approach would add considerable computational cost. 


\section{CONCLUSION}

Simultaneous PET-MR has shown potential for improving PET image quality by using MR information to address various degrading factors. In separate research works, MR-based motion compensation and MR-guided reconstruction have been demonstrated to improve PET image quality. In this work, these developments are integrated into a single PET-MR framework that produces high quality, diagnostic CMRA images alongside improved ${ }^{18} \mathrm{~F}-\mathrm{FDG}$ cardiac PET images. The proposed integrated PET image reconstruction framework improves image quality by including MR-based $\mu$-map alignment, respiratory motion correction and cardiac-gating and MR-based guidance of PET data.

The proposed framework was evaluated in terms of contrast and noise, and compared to a number of alternative reconstruction methods with each component of the PET reconstruction approach. The proposed method produced the highest contrast of all the methods, and significantly reduced image noise compared to a reference reconstruction that incorporates the motion compensation components of the framework without MR-guidance. In addition, applying the proposed framework to

${ }^{18}$ F-FDG PET data from cardiac patients demonstrated that the visual appearance of clinically relevant features, such as hypo-intense defects, are preserved. 


\section{DISCLOSURE}

No potential conflicts of interest relevant to this article exist.

\section{ACKNOWLEDGMENTS}

This work was supported by the following grants: (1) EPSRC EP/P032311/1, EP/P007619/1, EP/P001009/1; (2) BHF programme grant RG/20/1/34802 and (3) Wellcome/EPSRC Centre for Medical Engineering (WT 203148/Z/16/Z). This research was supported by the National Institute for Health Research (NIHR) Cardiovascular Health Technology Cooperative (HTC) and the Biomedical Research Centre based at Guy’s and St. Thomas' NHS Foundation Trust and King's College London. 


\section{KEY POINTS}

- QUESTION: What is the impact in image quality of incorporating MR-based motion compensation and anatomical guidance in cardiac PET image reconstruction?

- PERTINENT FINDINGS: The proposed method was tested in two cohorts of patients with myocardial uptake who underwent PET-MR examinations. The proposed PET reconstruction approach showed significant increase in myocardial-to-blood pool contrast compared to standard images, indicative of improved PET quantification. Compared to a converged PET image reconstruction method, inclusion of MR-guidance significantly reduced noise while preserving edges.

- IMPLICATIONS FOR PATIENT CARE: The improved myocardial PET image quality obtained by the proposed method has the potential to enhance diagnostic imaging by providing more accurate cardiac PET images. 


\section{REFERENCES}

1. Rischpler C, Siebermair J, Kessler L, et al. Cardiac PET/MRI: Current clinical status and future perspectives. Semin Nucl Med. 2020;50:260-269.

2. Vitadello T, Kunze KP, Nekolla SG, et al. Hybrid PET/MR imaging for the prediction of left ventricular recovery after percutaneous revascularisation of coronary chronic total occlusions. Eur J Nucl Med Mol Imaging. 2020.

3. Wicks EC, Menezes LJ, Barnes A, et al. Diagnostic accuracy and prognostic value of simultaneous hybrid 18F-fluorodeoxyglucose positron emission tomography/magnetic resonance imaging in cardiac sarcoidosis. Eur Hear J Cardiovasc Imaging. 2018;19:757-767.

4. Ouyang J, Li Q, El Fakhri G. Magnetic resonance-based motion correction for positron emission tomography imaging. Semin Nucl Med. 2013;43:60-67.

5. Robson PM, Dweck MR, Trivieri MG, et al. Coronary artery PET/MR imaging: Feasibility, limitations, and solutions. JACC Cardiovasc Imaging. 2017;10:1103-1112.

6. Kolbitsch C, Neji R, Fenchel M, Mallia A, Marsden P, Schaeffter T. Respiratory-resolved MRbased attenuation correction for motion-compensated cardiac PET-MR. Phys Med Biol. 2018;63:135008.

7. Manber R, Thielemans K, Hutton B, et al. Practical PET respiratory motion correction in clinical PET/MR. J Nucl Med. 2015;56:890-896.

8. Küstner T, Schwartz M, Martirosian P, et al. MR-based respiratory and cardiac motion correction for PET imaging. Med Image Anal. 2017;42:129-144.

9. Kolbitsch C, Neji R, Fenchel M, et al. Joint cardiac and respiratory motion estimation for motion-corrected cardiac PET-MR. Phys Med Biol. 2019;64:15007. 
10. Shepp LA, Vardi Y. Maximum likelihood reconstruction for emission tomography. IEEE Trans Med Imaging. 1982;1:113-122.

11. Schramm G, Holler M, Rezaei A, et al. Evaluation of parallel level sets and Bowsher's method as segmentation-free anatomical priors for time-of-flight PET reconstruction. IEEE Trans Med Imaging. 2018;37:590-603.

12. Bland J, Mehranian A, Belzunce MA, et al. Intercomparison of MR-informed PET image reconstruction methods. Med Phys. 2019;46:5055-5074.

13. Munoz C, Neji R, Cruz G, et al. Motion-corrected simultaneous cardiac positron emission tomography and coronary MR angiography with high acquisition efficiency. Magn Reson Med. 2018;79:339-350.

14. Qiao F, Pan T, Clark JW, Mawlawi OR. A motion-incorporated reconstruction method for gated PET studies. Phys Med Biol. 2006;51:3769-83.

15. Modat M, Ridgway GR, Taylor ZA, et al. Fast free-form deformation using graphics processing units. Comput Methods Programs Biomed. 2010;98:278-284.

16. Ellis S, Mallia A, McGinnity CJ, Cook GJR, Reader AJ. Multi-tracer guided PET image reconstruction. IEEE Trans Radiat Plasma Med Sci. 2018;2:499-509.

17. De Pierro AR. A modified expectation maximization algorithm for penalized likelihood estimation in emission tomography. IEEE Trans Med Imaging. 1995;14:132-137.

18. Reader AJ, Ellis S. Bootstrap-optimised regularised image reconstruction for emission tomography. IEEE Trans Med Imaging. 2020;39:2163-2175.

19. Munoz C, Kunze KP, Neji R, et al. Motion-corrected whole-heart PET-MR for the simultaneous visualisation of coronary artery integrity and myocardial viability: an initial clinical validation. Eur J Nucl Med Mol Imaging. 2018;45:1975-1986. 
20. Belzunce MA, Reader AJ. Assessment of the impact of modeling axial compression on PET image reconstruction. Med Phys. 2017;44:5172-5186.

21. Kikinis R, Pieper SD, Vosburgh KG. 3D Slicer: A platform for subject-specific image analysis, visualization, and clinical support. In: Intraoperative Imaging and Image-Guided Therapy. Springer, New York, NY; 2014:277-289.

22. Cerqueira MD, Weissman NJ, Dilsizian V, et al. Standardized Myocardial Segmentation and Nomenclature for Tomographic Imaging of the Heart. Circulation. 2002;105:539-542.

23. Thielemans K, Manjeshwar RM, Tao X, Asma E. Lesion detectability in motion compensated image reconstruction of respiratory gated PET/CT. In: 2006 IEEE Nuclear Science Symposium Conference Record. ; 2006:3278-3282.

24. Petibon Y, Ouyang J, Zhu X, et al. Cardiac motion compensation and resolution modeling in simultaneous $\{$ PET $\}-\{M R\}$ : a cardiac lesion detection study. Phys Med Biol. 2013;58:20852102.

25. De Pierro AR, Yamagishi MEB. Fast EM-like methods for maximum “a posteriori" estimates in emission tomography. IEEE Trans Med Imaging. 2001;20:280-288.

26. Schramm G, Rigie D, Vahle T, et al. Approximating anatomically-guided PET reconstruction in image space using a convolutional neural network. Neuroimage. 2021;224:117399.

27. Tsai Y, Bousse A, Ahn S, et al. Algorithms for solving misalignment issues in penalized PET/CT reconstruction using anatomical priors. In: 2018 IEEE Nuclear Science Symposium and Medical Imaging Conference Proceedings (NSS/MIC). ; 2018. 


\section{FIGURES}
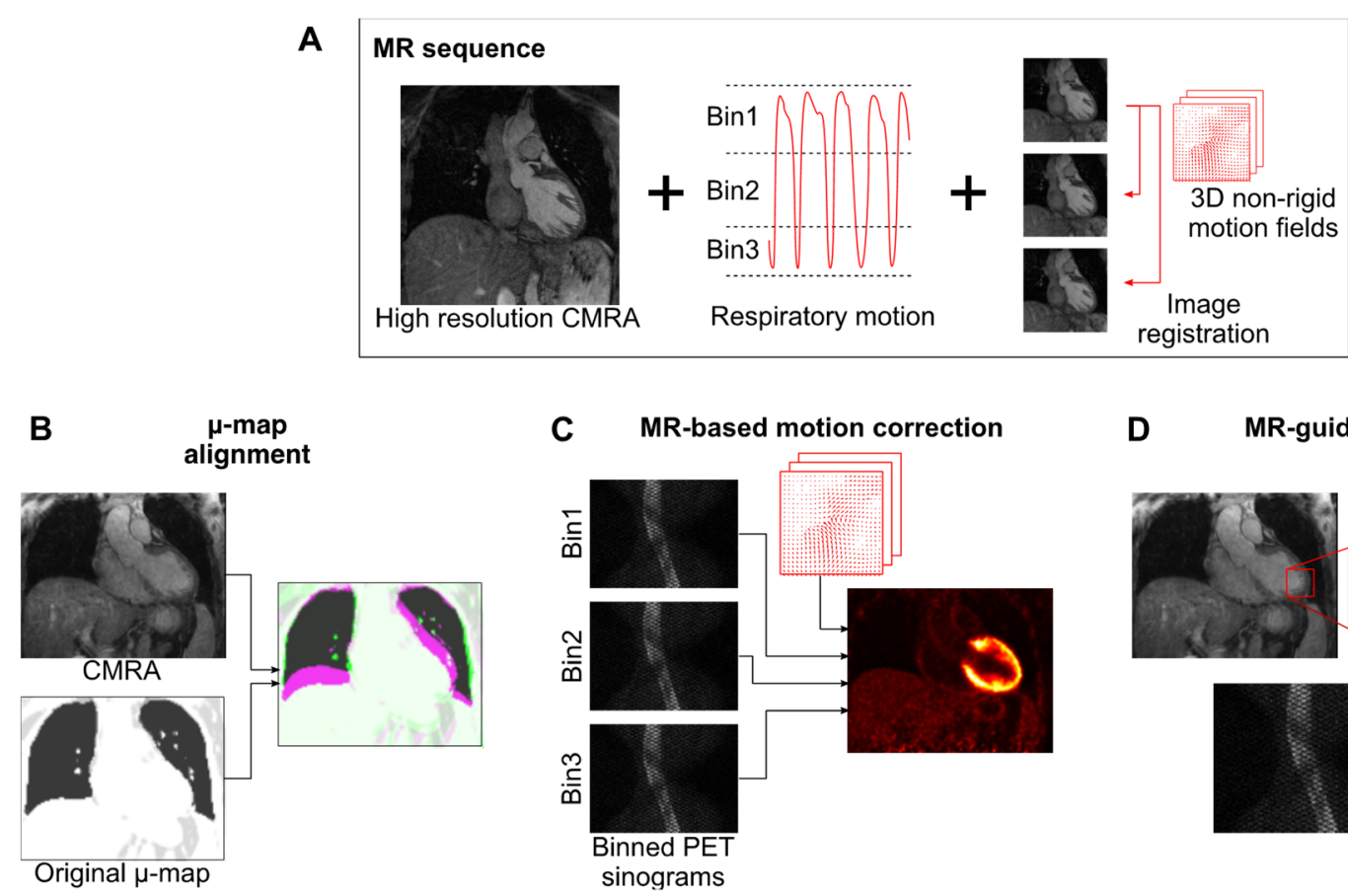

D MR-guided reconstruction

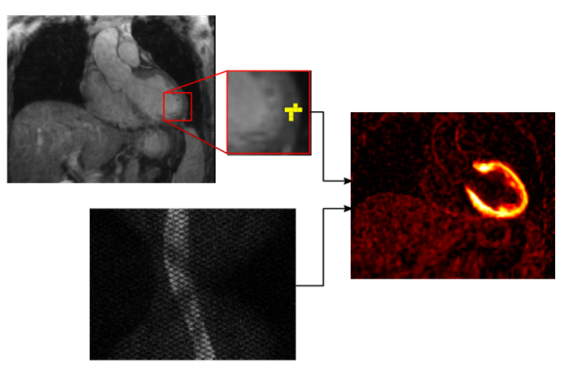

Figure 1: Overview of MR-based improvements for the proposed PET image reconstruction framework. (A) The MR sequence provides a high-quality end-expiration coronary MR angiography (CMRA) image, and respiratory motion information, which can be used to improve the PET image reconstruction by (B) aligning PET $\mu$-maps to the CMRA position, (C) performing MR-based motioncorrection, and (D) performing MR-guided PET image reconstruction. 


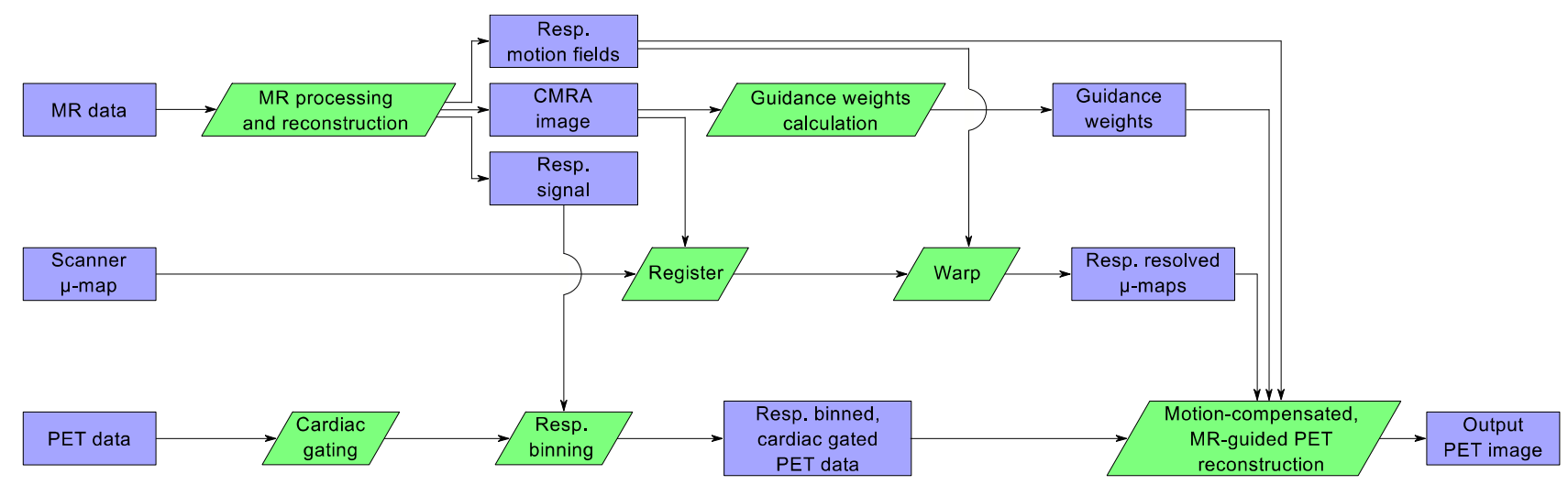

Figure 2: Flowchart of the proposed PET-MR image reconstruction pipeline.
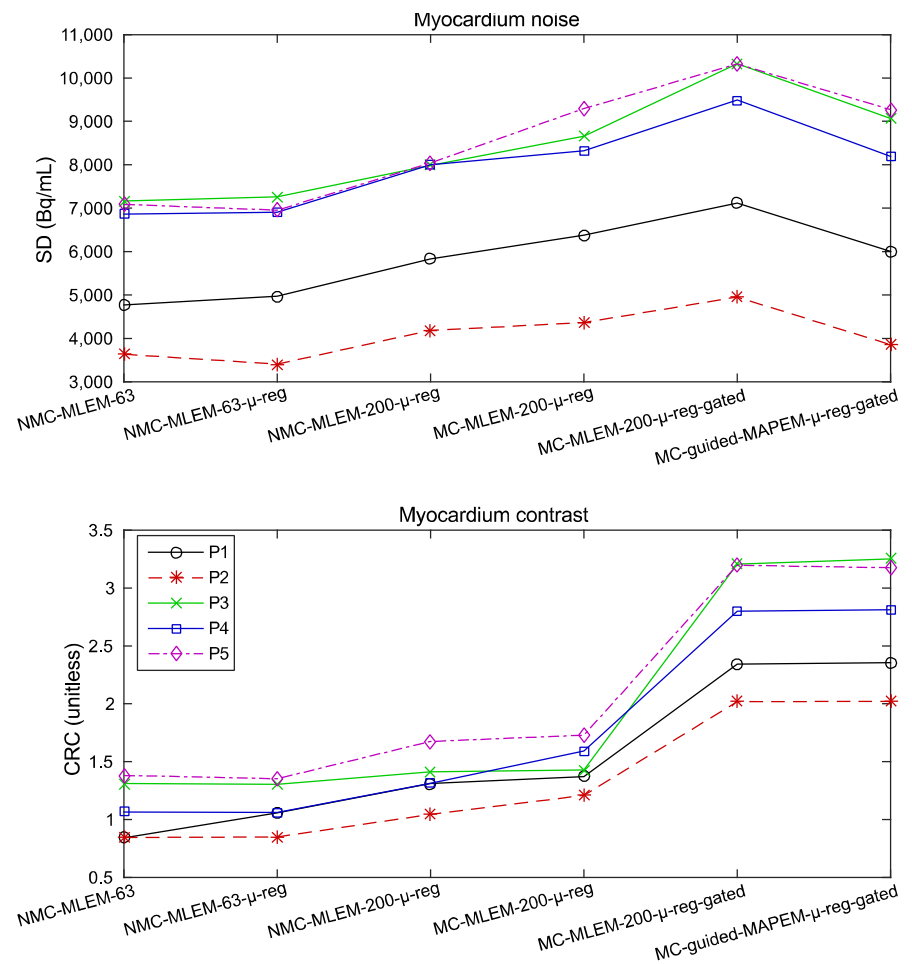

Figure 3: Image noise and contrast in five oncology patients for each PET image reconstruction method. The proposed method provides the highest myocardium-to-blood pool contrast levels (CRC) in all cases, while avoiding high noise levels (SD) of unregularised PET image reconstruction. 


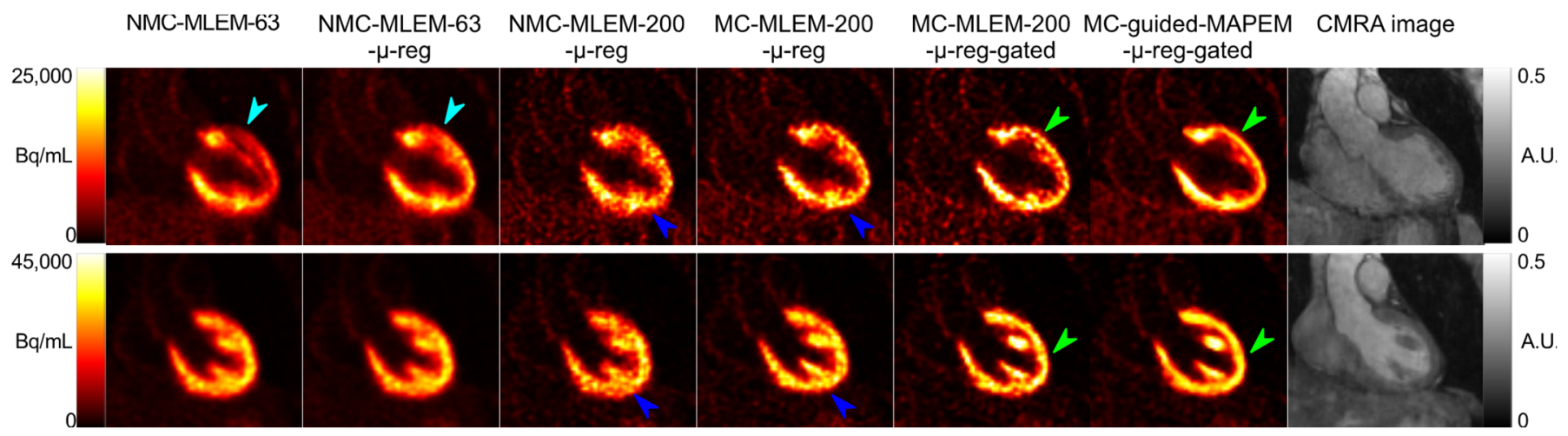

Figure 4: Reconstructed images for each comparative method for two representative oncology patients (one per row). Cyan arrows indicate a myocardial defect-mimicking attenuation artefact which is removed by aligning the $\mu$-map using the CMRA image. Blue arrows highlight improved local contrast when using motion-corrected PET image reconstruction. Green arrows show reduced noise and improved sharpness when combining motion compensation with MR-guided PET image reconstruction. 


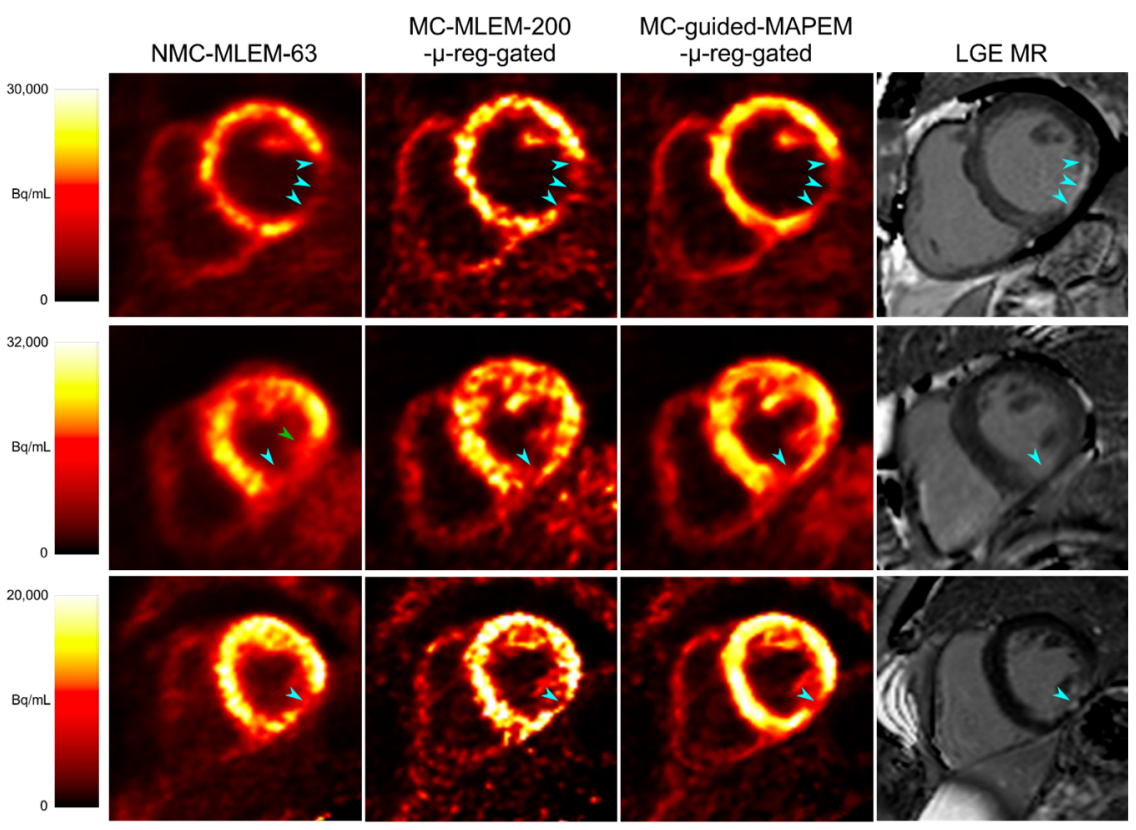

Figure 5: Example short-axis view of the reconstructed ${ }^{18}$ F-FDG PET images for three selected CTO patients, and corresponding late-gadolinium enhancement (LGE) MR images, showing the extent of myocardial scarring. The proposed method improves image quality while maintaining the appearance of ${ }^{18}$ F-FDG hypo-intense defects (cyan arrows). MC-MLEM-200- $\mu$-reg-gated images are shown to distinguish the effects of motion compensation and guidance. In some cases, the uncorrected PET images falsely depict the defect as more extensive than it actually is (green arrow, row 2). Note that LGE images are shown only for comparison and did not provide any information for the guided PET reconstructions, which instead used high-resolution CMRA images. 
TABLES

\begin{tabular}{lcccc}
\hline CTO+Onco $(n=15)$ & $\overline{\mathrm{CRC}}($ unitless) & $p$-value & $\overline{\mathrm{SD}}(\mathrm{Bq} / \mathrm{mL})$ & $p$-value \\
\hline NMC-MLEM-63 & $0.92 \pm 0.30$ & & $5443 \pm 1636$ & \\
NMC-MLEM-63- $\mu$-reg & $0.96 \pm 0.30$ & $0.033(\uparrow)$ & $5512 \pm 1660$ & $0.20(\uparrow)$ \\
NMC-MLEM-200- $\mu$-reg & $1.23 \pm 0.39$ & $8.2 \times 10^{-7}(\uparrow)$ & $6565 \pm 1805$ & $1.2 \times 10^{-8}(\uparrow)$ \\
MC-MLEM-200- $\mu$-reg & $1.34 \pm 0.44$ & $0.078(\uparrow)$ & $7033 \pm 1974$ & $5.0 \times 10^{-5}(\uparrow)$ \\
MC-MLEM-200- $\mu$-reg-gated & $2.22 \pm 0.72$ & $1.1 \times 10^{-5}(\uparrow)$ & $7752 \pm 2302$ & $3.7 \times 10^{-5}(\uparrow)$ \\
MC-guided-MAPEM- $\mu$-reg-gated & $2.24 \pm 0.73$ & $0.0026(\uparrow)$ & $6504 \pm 2237$ & $1.7 \times 10^{-8}(\downarrow)$ \\
\hline * & & & \\
\hline
\end{tabular}

Table 1: Statistical analysis of the effect of the comparative methods in terms of CRC and SD for the combined patient cohort (CTO+Onco). $p$-values from two-tailed paired $t$-tests are shown, along with the sign of the change ( $\uparrow$ for positive, $\downarrow$ for negative), comparing the method in each row with the previous row. Final row $(*)$ shows $p$-values comparing the proposed method with the clinical standard NMC-MLEM-63. 
GRAPHICAL ABSTRACT

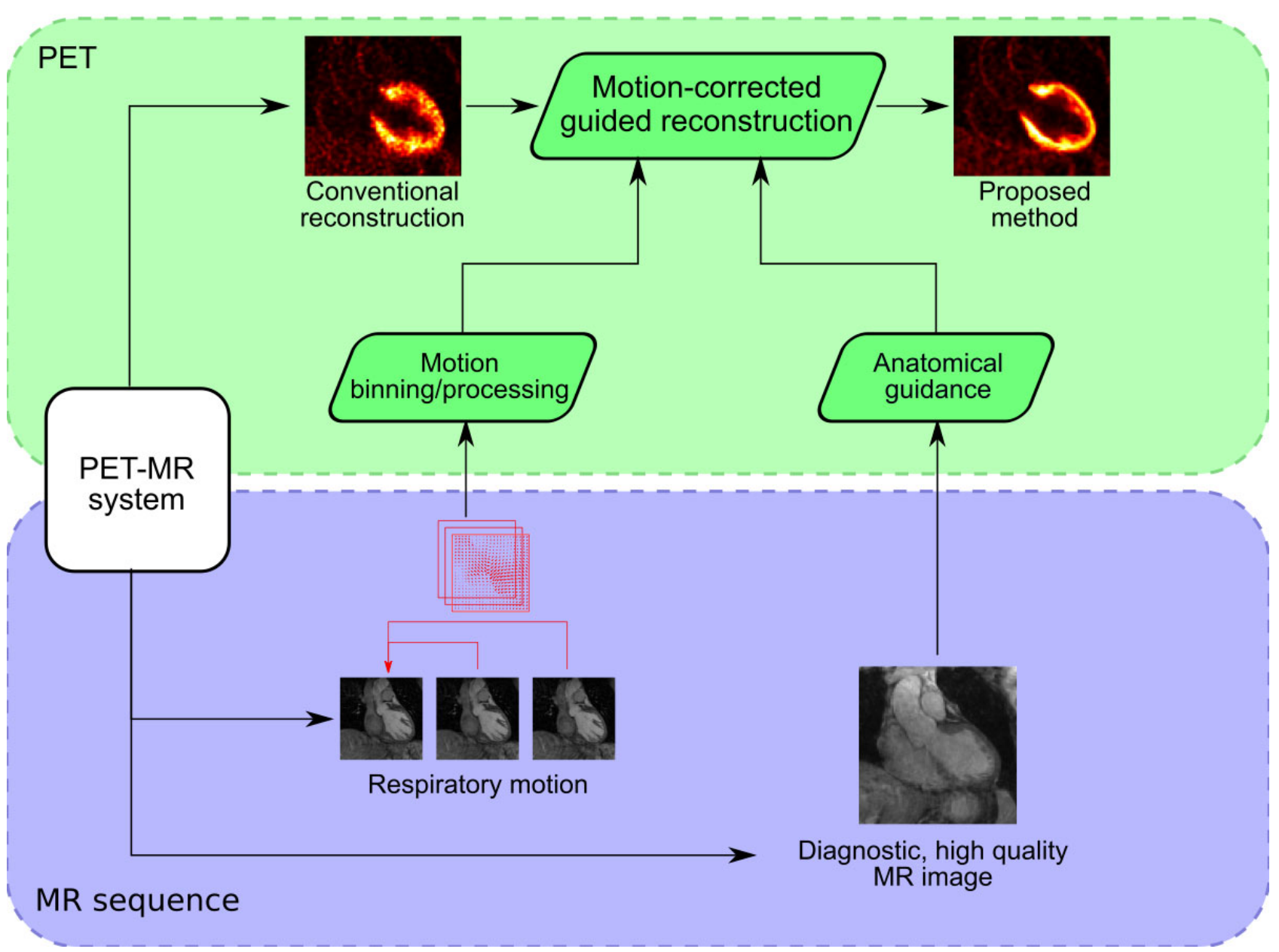




\section{SUPPLEMENTARY MATERIAL}

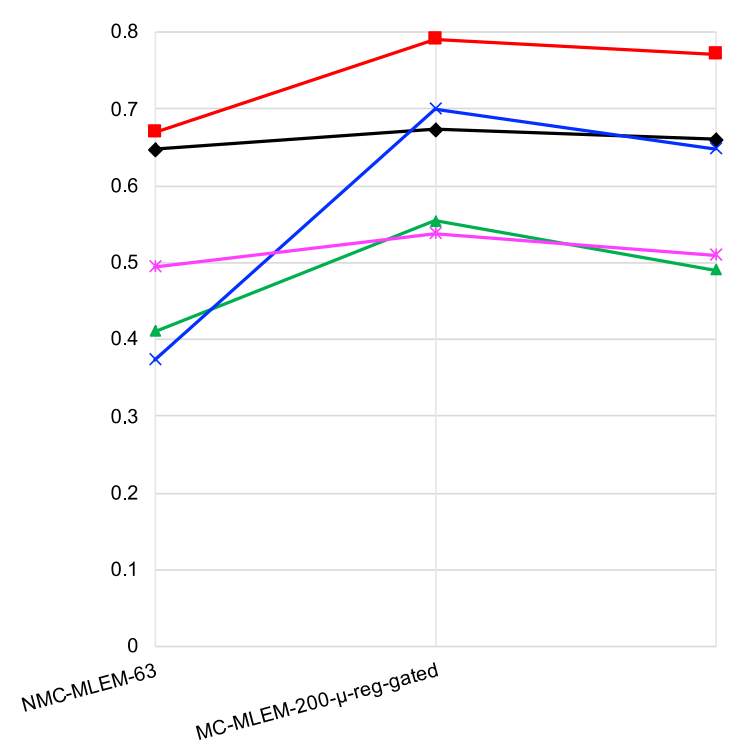

Supplementary Figure 1. Contrast recovery coefficient (CRC) and contrast-to-noise ratio (CNR) between healthy myocardium and transmural viability defects for five cardiac patients, for the NMC-MLEM-63, MC-MLEM-200- $\mu$-reg-gated and proposed anatomically guided motioncompensated method (MC-guided-MAPEM- $\mu$-reg-gated). The proposed method improves CRC by $18.6 \%$ on average and CNR by $47.7 \%$ compared to the conventional reconstruction (NMCMLEM-63). 

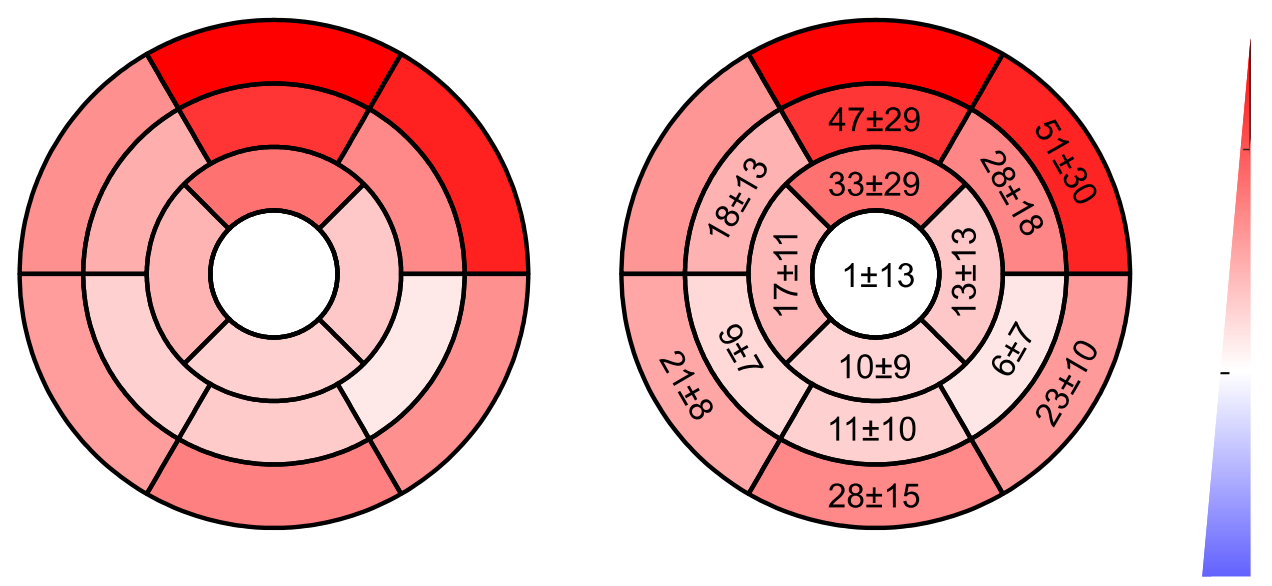

Supplementary Figure 2. 17 segment analysis showing the relative difference in PET uptake values between (a) MC-MLEM-200- $\mu$-reg-gated and NMC-MLEM-63, and (b) the proposed MC-guidedMAPEM- $\mu$-reg-gated method and NMC-MLEM-63. While an overall increase of $\sim 17 \pm 9 \%$ was observed for both MC-MLEM-200- $\mu$-reg-gated and MC-guided-MAPEM- $\mu$-reg-gated methods, some areas of the left ventricle myocardium show a significantly larger increase than others, with an $\sim 15 \%$ increase at the apical segments to over $60 \%$ increase in the basal anterior segment. 


\begin{tabular}{lcc}
\hline & $\overline{\text { FWHM }}$ & $p$-value \\
\hline NMC-MLEM-63 & $3.81 \pm 0.95$ & - \\
NMC-MLEM-63- $\mu$-reg & $3.73 \pm 0.93$ & $0.097(\downarrow)$ \\
NMC-MLEM-200- $\mu$-reg & $3.54 \pm 1.01$ & $0.008(\downarrow)$ \\
MC-MLEM-200- $\mu$-reg & $3.35 \pm 0.90$ & $0.064(\downarrow)$ \\
MC-MLEM-200- $\mu$-reg-gated & $2.74 \pm 0.73$ & $1.44 \times 10^{-5}(\downarrow)$ \\
MC-guided-MAPEM- $\mu$-reg-gated & $2.81 \pm 0.60$ & $0.268(\uparrow)$ \\
\hline$*$ & - & $9.48 \times 10^{-8}(\downarrow)$ \\
\hline
\end{tabular}

Supplementary Table 1: Statistical analysis of the effect of the comparative methods in terms of myocardial sharpness. For each method, the mean FWHM $(\overline{\mathrm{FWHM}})$ over three profiles selected for each oncology patient are listed, along with an uncertainty given by the standard deviation over all profiles. $p$-values from two-tailed paired $t$-tests are shown, along with the sign of the change $(\uparrow$ for positive, $\downarrow$ for negative), comparing the method in each row with the previous row. Final row (*) shows $p$-values comparing the proposed method with the clinical standard NMC-MLEM-63. 


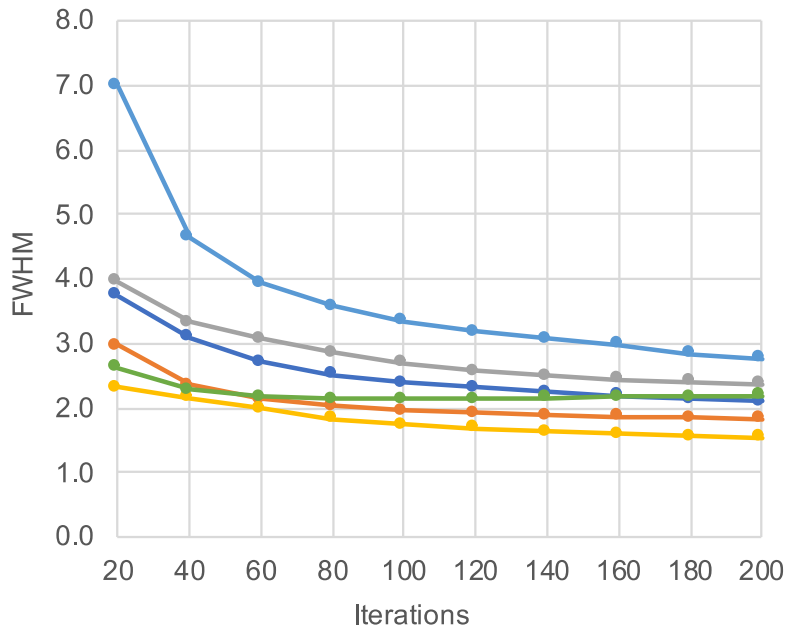

(a) MC-MLEM-200- $\mu$-reg-gated

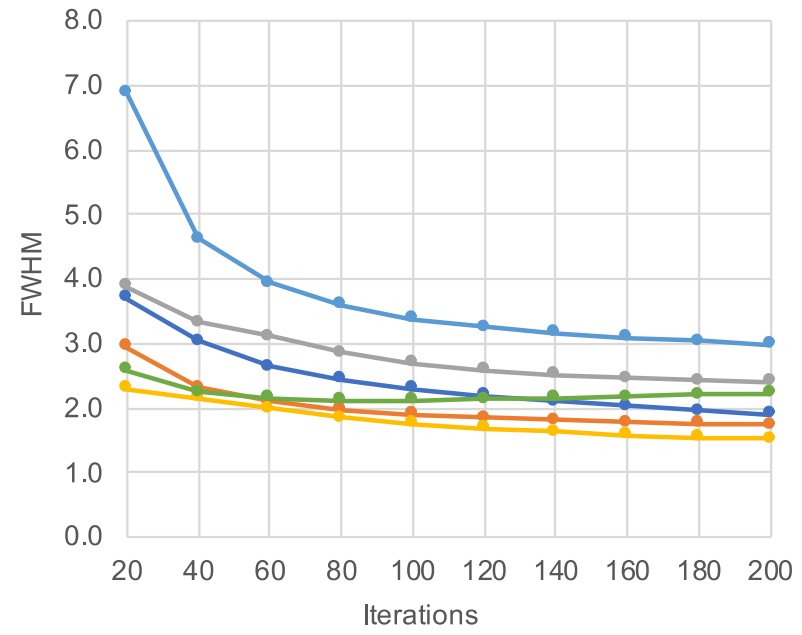

(b) MC-guided-MAPEM- $\mu$-reg-gated

Figure R1 (for review only). Myocardial sharpness measured as full-width at half-maximum (FWHM) for selected profiles through the left ventricle myocardium including apical, mid, and basal regions. in a representative patient for (a) MC-MLEM-200- $\mu$-reg-gated and (b) the proposed MC-guided-MAPEM- $\mu$-reg-gated method as a function of the number of iterations. In both cases, the FWHM consistently decreases with increasing number of iterations, suggesting that inconsistencies in the motion estimation step do not distort the resulting images. 